\title{
Abordagem Sistémica da Tolerância ao Exercício: Um Caso para a Modelação Matemática
}

\section{Systemic Approach Towards Understanding Exercise Tolerance: The Case for a Mathematical Model Application}

João Beckert ${ }^{1,2}$, João Pedro Mendes ${ }^{3}$, Francisco Alves ${ }^{4}$, Nuno Neuparth ${ }^{1}$

Autor Correspondente:

\section{RESUMO}

A prescrição do exercício pressupõe que se antecipe a tolerância ao esforço de cada indivíduo. Os erros e imprecisões na determinação da intensidade a prescrever e a complexidade da resposta fisiológica ao exercício comprometem a aplicação das orientações genéricas.

A modelação matemática é uma ferramenta para a previsão da resposta fisiológica ao exercício e, por isso, pode tornar-se útil para melhorar a prescrição do exercício.

Neste artigo é apresentado o modelo matemático adaptado e desenvolvido pelos autores. É feita referência às perspetivas de aplicação destas metodologias de investigação teórica, tanto no domínio da fisiologia do exercício como no da saúde das populações. Conclui-se ser oportuno a criação de um núcleo de investigação em medicina desportiva com inclusão da modelação matemática em sistemas dinâmicos.

PALAVRAS-CHAVE: Análise de Sistemas; Medicina Desportiva; Modelos Teóricos; Tolerância ao Exercício

\section{ABSTRACT}

To prescribe exercise, one must anticipate each individual's exercise tolerance. The errors and imprecisions associated with the determining of the prescribed intensity, as well as the complexity of the physiological response, compromise the application of generic guidance.

Mathematical modelling is a tool for predicting the physiological response to exercise and can, therefore, be useful for prescribing exercise.

The mathematical model adapted and developed by the authors is presented and explained in the article. Also referred are the perspectives towards the application of these theoretical investigation methodologies, either in the exercise physiology domain, as in the populations' health. As a conclusion, it is timely that the creation of a research nucleus in Sports Medicine includes mathematical modelling in systems dynamics.

KEYWORDS: Exercise Tolerance; Models, Theoretical; Sports Medicine; Systems Analysis

1.CEDOC - NOVA Medical School, UNL, Lisboa, Portugal. 2. Centro de Alto Rendimento do Jamor, IPDJ, Lisboa, Portugal. 3. CENTEC - IST-UL, Lisboa, Portugal. 4. CIPER, FMH-UL, Lisboa, Portugal.

Recebido: 23/05/2017 - Aceite: 29/05/2017 


\section{INTRODUÇÃO}

A atividade física tem um impacto benéfico na saúde das populações e existem programas para a sua promoção, com medidas transversais dos setores da saúde, educação e desporto. Os programas de promoção da atividade física estão assentes em recomendações genéricas de prescrição do exercício, que tomam em consideração os domínios de intensidade estabelecidos pela relação entre a intensidade do exercício e a tolerância ao esforço.

A determinação dos domínios de intensidade como moderado, pesado e severo acarreta erros e imprecisões que restringem a aplicabilidade das prescrições genéricas, sobretudo nos casos extremos, como os indivíduos com acentuada limitação da capacidade funcional e os atletas de alto rendimento. Deste modo, torna-se necessário desenvolver instrumentos capazes de melhorar a previsão da resposta fisiológica ao exercício e, portanto, da tolerância ao exercício.

O nosso objetivo é i. Apresentar o modelo matemático da resposta fisiológica ao exercício, fruto da colaboração de 3 centros de investigação, ii. Apresentar os fundamentos da abordagem em sistemas dinâmicos, iii. Enquadrar historicamente estes instrumentos de investigação teórica, iv. Explicar a construção do modelo desenvolvido e $\mathbf{v}$. Referir as perspetivas de aplicação e desenvolvimento.

\section{APRESENTAÇÃO DO MODELO DA RESPOSTA FISIOLÓGICA AO EXERCÍCIO}

O modelo matemático desenvolvido permite prever a resposta fisiológica ao exercício e explica as interações mútuas entre as variáveis, em diferentes condições de exercício e de ambiente, com base nos mecanismos de regulação energética e de regulação da perfusão muscular.

Dada a natureza sistémica da resposta fisiológica, com relações não lineares e interações mútuas entre as variáveis, ciclos de realimentação e atrasos no tempo, a modelação matemática em sistemas dinâmicos foi a abordagem escolhida. $\bigcirc$ modelo da resposta fisiológica ao exercício é construído com base nos princípios da física e da fisiologia e utiliza a matemática como linguagem para exprimir as propriedades identificadas experimentalmente.

O modelo matemático da resposta fisiológica ao exercício foi adaptado para a plataforma informática escolhida, com base nas propostas de outros autores e incorpora as propostas inovadoras do cálculo do rendimento e da simulação do Q ${ }_{m}$ em função da taxa metabólica, expressa como V·O_2. No cálculo do rendimento, o modelo tem em conta os contributos aeróbio e não aeróbio e representa como um feedback positivo o aumento do consumo de energia, quando a produção de energia aeróbia não satisfaz as necessidades acrescidas de ATP, como ocorre nos domínios de intensidade pesado e severo e nas transições. Pela simulação do $Q_{m}^{*}$ e das variáveis relacionadas com o transporte de $\mathrm{O}_{\text {_2}}$, o modelo permite identificar quando a 【PO』_2 nos tecidos é inferior ao valor crítico para o metabolismo aeróbio e, assim, esclarecer porque, em altitude, o exercício de intensidade moderada pode apresentar características de exercício pesado, sem aumento do $\mathrm{V}^{\circ} \mathrm{O} \_2$.

Os resultados das simulações já efetuadas indicam que o modelo matemático desenvolvido é adequado para a previsão da resposta fisiológica ao exercício e tem, portanto, interesse na prescrição do exercício e é útil na formação médica.

\section{NATUREZA SISTÉMICA DA RESPOSTA FISIOLÓGICA}

Os sistemas fisiológicos caracterizam-se pela existência de múltiplos processos de controlo que asseguram a manutenção das condições do meio interior em valores compatíveis com a vida, como foi referido no século XIX por Claude Bernard, citado por Noble. ${ }^{1} O$ conceito de millieu intérieur, de Claude Bernard, é o princípio subjacente ao conceito de homeostase apresentado por Walter Cannon em 1932.

Os processos de controlo e a não linearidade dos sistemas biológicos estão na base da sua adaptabilidade e auto-organização. 1,2 Sterman³ realça que "a complexidade dos sistemas não provém somente do elevado número de constituintes, dos diferentes estados possíveis do sistema ou das múltiplas combinações possíveis, i.e., de uma complexidade combinatória. A complexidade provém da dinâmica relativa às interações dos seus agentes no tempo e da forma como estas determinam o comportamento desse sistema (i.e. de uma complexidade dinâmica)".

Para Gallaher, ${ }^{4}$ diversos problemas biomédicos, incluindo diabetes, hipertensão e tolerância medicamentosa são fundamentalmente problemas de sistemas de controlo biológico, em que a modelação e a simulação são ferramentas eficazes para o seu estudo sistemático.

A explicação da resposta fisiológica ao exercício é um problema de natureza sistémica, com inerente complexidade dinâmica, em que é conveniente recorrer à representação destes comportamentos com recurso a modelos matemáticos de sistemas dinâmicos. 
Os modelos matemáticos baseados em sistemas dinâmicos são diferentes dos modelos matemáticos baseados na estatística porque envolvem uma componente temporal que permite a simulação do comportamento. ${ }^{5}$ Estes modelos são adequados para lidar com sistemas descritos por um conjunto de séries temporais, como é o caso da caracterização da resposta fisiológica ao exercício. Além disso, nos modelos em sistemas dinâmicos, as relações entre as diferentes variáveis de interesse estão fundamentadas nos princípios da física e da química como a lei da conservação de massa, a lei da conservação de energia, e as leis fenomenológicas dos processos de transporte e difusão e também permitem representar comportamentos em diferentes escalas. Para An et al, ${ }^{5}$ os modelos nas escalas dos tecidos, órgãos e sistemas são os mais apropriados porque constituem uma ponte entre a ciência básica, os dados experimentais reducionistas e o fenómeno clínico.

Wellstead et al, ${ }^{6}$ consideram que a aplicabilidade dos modelos em sistemas dinâmicos abrange escalas mais vastas, como as dos processos metabólicos, vias de sinalização e sistemas de regulação de genes.

\section{MODELAÇÃO MATEMÁTICA EM FISIOLOGIA \\ - ENQUADRAMENTO HISTÓRICO}

Em 1965, Arthur Guyton foi um dos autores do artigo A Mathematical Model of Respiratory Control System.7 A representação do sistema circulatório num modelo de dois compartimentos com recurso a relações lineares entre as variáveis, proposta por Guyton em 1966, é considerada um contributo significativo para a compreensão da regulação da circulação Keener e Sneyd. ${ }^{8}$ A perspetiva da modelação em fisiologia médica é patente nas obras do autor, utilizadas com frequência no ensino da fisiologia médica.

Grodins et al, 9 publicaram um modelo de três compartimentos (pulmão, cérebro e outros tecidos), que descreve o comportamento do O_2, do «CO』_2 e do N_2 e que é referência para o desenvolvimento de outros modelos.

Um dos primeiros modelos que descreve os processos energéticos durante o exercício foi publicado por Margaria em 1976,10 como um modelo hidráulico qualitativo, mas só cerca de uma década depois foi publicada a sua resolução algébrica. ${ }^{11}$

Wilson e coautores, apresentaram uma proposta do controlo da respiração mitocondrial em que a taxa de respiração mitocondrial é dependente do rácio [ATP]/ [ADP] [Pi] e do nível de redução do citocromo c (relati- vo ao rácio $\left.\left[\llbracket N A D \rrbracket^{\wedge}+\right] /[\mathrm{NADH}]\right) .{ }^{12,13}$ Os autores deram continuidade ao estudo da regulação energética e, mais tarde, incluíram o parâmetro da 【PO』_2 no seu modelo matemático e confirmaram a dependência da fosforilação oxidativa da concentração de O_2, experimentalmente avaliada em preparação isolada de mitocôndrias de rato.

Em 1990, Linnarsson, ${ }^{14}$ apresentou o contributo da engenheira de sistemas para a compreensão da resposta bioenergética na transição do repouso para o exercício. No mesmo ano, diversos autores apresentaram contributos para o estudo dos mecanismos de controlo dos gases ventilatórios, ${ }^{15}$ propondo orientações para a construção de modelos da dinâmica dos gases ${ }^{16}$ e indicando os determinantes fisiológicos da cinética do $02 .{ }^{17}$

Barstow et al, ${ }^{18}$ em 1990, publicaram um modelo de três compartimentos condensados (sistema pulmonar, circulatório e músculo em exercício) e demonstraram como a interação entre o sistema circulatório e respiratório explica as características da fase inicial (fase I, cardiodinâmica) e da fase intermédia (fase II), na transição do repouso para o exercício.

Cabrera et al, ${ }^{19}$ em 1998, apresentaram um modelo matemático do balanço de massa e das características da cinética do glicogénio, glicose, piruvato, lactato, $\mathrm{O}_{-} 2$, 【CO』_2 e ATP entre o compartimento esplâncnico, muscular e "outros tecidos". O modelo testa a regulação do lactato em situação de hipoxia, em repouso. Em 1999, o mesmo grupo de autores apresentou a análise do metabolismo do lactato em exercício. ${ }^{20}$ Lai et al, ${ }^{21-23}$ do mesmo grupo de investigação, apresentaram versões meIhoradas de modelos de transporte e utilização de O_2, incluindo a componente de regulação metabólica e do fluxo, com utilização da monitorização da oximetria do tecido muscular em situação de exercício de intensidade moderada, pesada e severa.

Mader, ${ }^{24}$ em 2003, apresentou um modelo do controlo metabólico da produção oxidativa e glicolítica de ATP, como função do consumo de ATP em repouso e em exercício. O modelo de Mader utiliza um sistema de duas equações diferenciais não lineares de primeira ordem e 29 equações algébricas. Este modelo é complementado com a distribuição e eliminação do lactato no compartimento muscular e sanguíneo. $\bigcirc$ modelo adequa-se à representação dos três sistemas energéticos (aeróbio, anaeróbio láctico e anaeróbio aláctico).

Moxnes et al, ${ }^{25}$ em 2012, recorreram à modelação matemática para testar a relação entre a potência e capacidade dos sistemas energéticos aeróbio e anaeróbio láctico e a potência desenvolvida durante a atividade 
física. Moxnes e Sandbakk, ${ }^{26}$ desenvolveram um modelo de produção e remoção do lactato no compartimento muscular e sanguíneo, validado com um sujeito, e relatam divergências nas simulações em exercício inferiores a $0,5 \mathrm{mmol} \cdot \mathrm{L}^{\wedge}(-1)$.

\section{CONSTRUÇÃO DO MODELO}

O nosso modelo representa o musculoesquelético e os limites do modelo identificam a fronteira entre os componentes excluídos e os incluídos. A estrutura do modelo consiste no conjunto de relações entre as variáveis de estado que são consideradas como o nível de um reservatório controlado por taxas de entrada e saída. Graficamente, nestes diagramas os reservatórios são representados como retângulos e as entradas e saídas como "condutas".

Para além das variáveis de nível, que indicam a acumulação de uma quantidade num reservatório ao longo do tempo e cujo valor instantâneo corresponde à quantidade armazenada no reservatório nesse instante, o modelo utiliza também as variáveis de fluxo, cuja variação ao longo do tempo representa a velocidade com que a quantidade entra (ou sai) dos reservatórios. Os fluxos fazem baixar ou subir os níveis dos reservatórios e exprimem-se em quantidade por unidade de tempo. A evolução do comportamento do sistema depende das taxas de variação (fluxos) de entrada e saída dos reservatórios.

O valor do nível no momento t_ $(n+1)$ é dado pelo valor do reservatório no momento anterior t_n, adicionado ao valor expresso pelas diferenças de fluxo nesse intervalo de tempo. Desta forma, diz-se que "as taxas de variação são integradas nos reservatórios". Nos modelos matemáticos de sistemas dinâmicos, estas relações são equações integrais não lineares, expressas pela relação: nível $(t)=\int_{\_} 0^{\wedge} t:: \llbracket($ taxa de entrada $(t)$-taxa de saída $(t)) \cdot d t \rrbracket$.

O valor do integral, no domínio do tempo, é obtido por métodos numéricos durante a simulação. A interface gráfica do software de sistemas dinâmicos Vensim ${ }^{\circledR}$ permite obter resultados de simulações sem que o utilizador tenha de lidar com as complexidades matemáticas destas equações integrais (ou diferenciais).

O tempo entre as entradas e as saídas nos reservatórios corresponde ao tempo de permanência nos reservatórios e condiciona o efeito tampão (buffer).

Tal como acontece no sistema real, os "tempos" de circulação ou de permanência desempenham um papel importante no comportamento do modelo, nomeadamente na descrição dos fenómenos de ampliação ou inibição. O tempo de trânsito ou circulação corresponde ao tempo de ligação entre os reservatórios e é designado por atraso, atraso de condução ou time delay.
Os ciclos de realimentação, de retrocontrolo ou de feedback desempenham um papel determinante no comportamento do sistema. Os ciclos de realimentação positiva são responsáveis pela dinâmica de alteração de um sistema, como o crescimento e evolução, por exemplo. Os ciclos de realimentação negativa são responsáveis pela regulação e a estabilidade, pelo que estabelecem a homeostasia e a autoconservação.

\section{MECANISMO DE CONTROLO DO ATP E REGULAÇÃO ENERGÉTICA}

modelo inclui uma equação diferencial publicada por Mader, ${ }^{24}$ que explicou o controlo metabólico da produção de ATP como função da utilização do ATP, afirmando que a taxa de mudança dos fosfatos de alta energia no citosol determina a fosforilação de ATP (proveniente da fosforilação oxidativa ou da glicólise anaeróbia) para compensar o ATP utilizado, em repouso ou du-

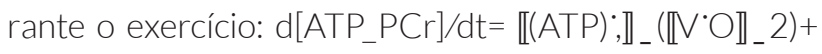
$\llbracket(A T P) ; \rrbracket_{-}\left(L_{-} a\right)^{\circ}-\left(\llbracket(A T P) ; \rrbracket_{-} r e p o u s o+\llbracket(A T P) ;, \rrbracket_{-} W\right)[E q 1]$.

\section{MECANISMO DE CONTROLO DO OXIGÉNIO NO TECIDO MUSCULAR}

Por seu lado, Lai et al, ${ }^{21,23}$ descreveram o controlo do conteúdo de oxigénio no tecido muscular durante um intervalo de tempo como o balanço entre o aporte de oxigénio pelo fluxo arterial, a taxa de utilização de oxigénio $(\mathbb{V} \cdot \bigcirc \rrbracket$ _ 2) e o retorno de oxigénio no sangue ve-

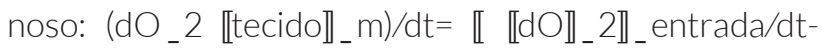

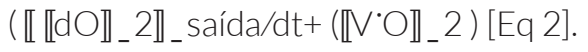

\section{OUTRAS RELAÇÕES FENOMENOLÓGICAS ENTRE OS CONSTITUINTES DO MODELO}

Depois da identificação dos mecanismos de controlo dos fosfatos de alta energia [Eq 1] e do conteúdo de oxigénio no tecido muscular [Eq 2], estes são montados em conjunto com as relações fenomenológicas, expressas por equações. As relações fenomenológicas são as relações de constituição que traduzem, em conjunto com as equações diferenciais [1] e [2], a estrutura matemática do modelo. As relações fenomenológicas foram organizadas em grupos e cada um dos grupos suporta a solução de problemas específicos de cálculo (Fig. 1):

Fosfatos de alta energia - Calcula a concentração dos fosfatos de energia do citosol, baseado nas equações (5) a (8) de Mader $^{24}$ que utilizam [PCr],[ATP],[ADP],[AMP] e 
$\left[\mathrm{H}^{\wedge}+\right]$ e as respetivas constantes de equilíbrio de reação.

Glicólise anaeróbia - Calcula o valor da glicólise em estado estacionário ((L_a )’ss), baseado nas equações (14) a (16) de Mader $^{24}$ que utilizam [ADP],[AMP], $\left[\mathrm{H}^{\wedge}+\right]$, as constantes de equilíbrio correspondentes, e (L_a )'máx., em que (L_a )'máx. é a taxa máxima de glicólise anaeróbia, expressa como uma taxa de produção de ácido láctico.

Fosforilação oxidativa - Calcula o valor do consumo de O_(2 ) necessário para exercício ( $\mathbb{V} \cdot \mathrm{O} \_2$ ss), baseado no sistema de equações (9) de Mader (24), que utilizam V•O_2 "máx." e [ADP] recorrendo à relação de Hill associada à cinética de Michaelis-Menton.
Lactato - Calcula as concentrações de lactato no músculo e no sangue arterial com base na taxa de formação de lactato (corrigida para o pH), no (L_a )'ss e na eliminação de lactato por oxidação e resíntese de glicogénio. ${ }^{24}$

Saturação de hemoglobina - Calcula as saturações de hemoglobina no sangue arterializado ( $\llbracket \mathrm{SaO} \_$2) e no capilar muscular 【(ScapO』_2) e a saturação de mioglobina no tecido muscular (S_m O_2Mb) a partir da pressão parcial de oxigénio ( $\mathrm{PaO}$ _2), usando a relação de Hill com parâmetros propostos por Keener e Sneyd. ${ }^{8}$

Difusão de oxigénio - Calcula o oxigénio dissolvido nos capilares e no tecido muscular com a equação (3) de Lai et al. ${ }^{21}$

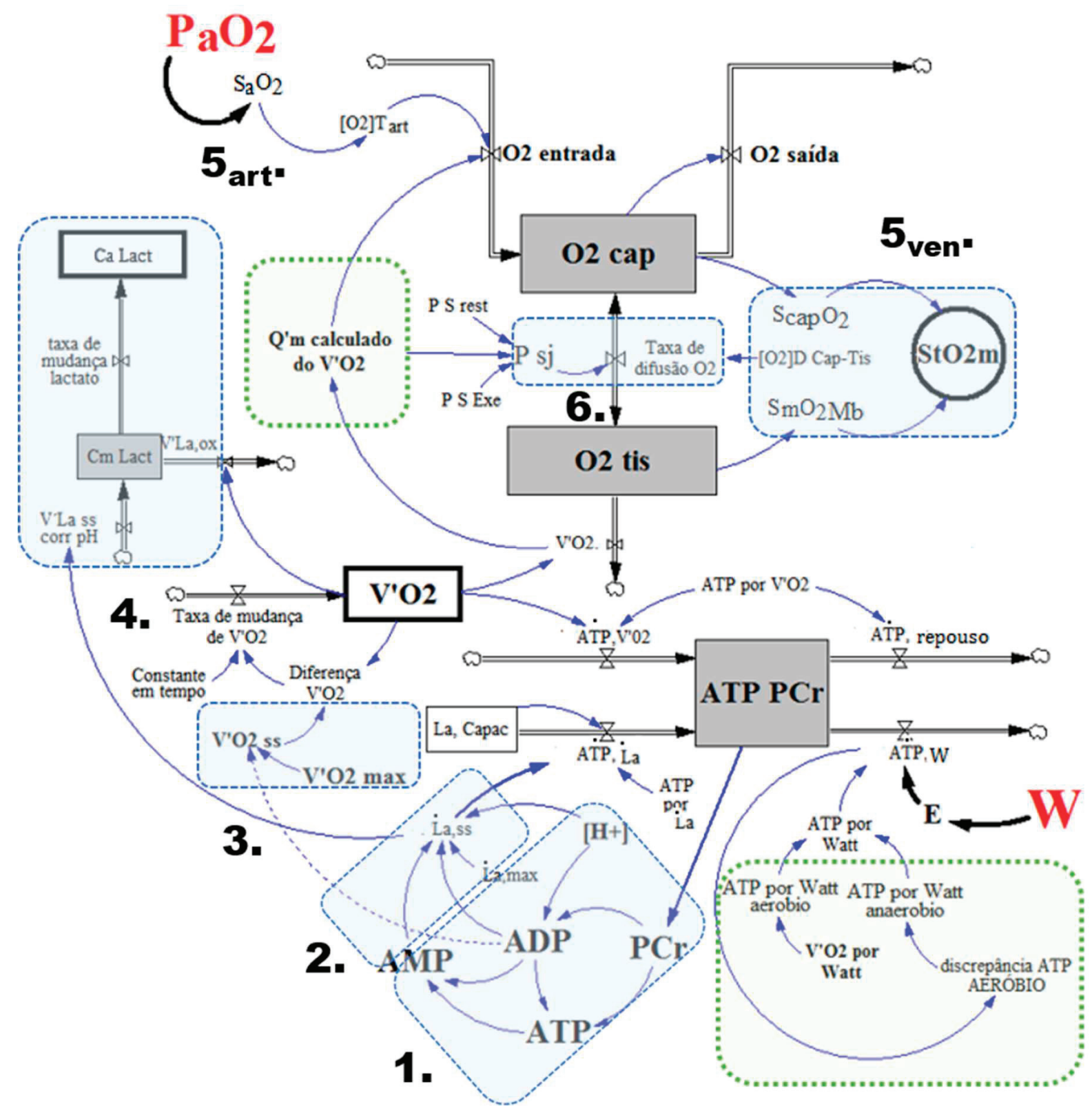

FIGURA 1. Representação simplificada do modelo com uso da interface gráfica do software de sistemas dinâmicos utilizado (Vensim ${ }^{\circledR}$ DSS). O diagrama mostra as variáveis externas $\mathrm{We}$ e $\mathrm{P}_{\mathrm{a}} \mathrm{O}_{2}$ (vermelho) e as variáveis medidas em laboratório (caixa em negrito para V $\mathrm{O}_{-} 2$ e Ca Lact e círculo em negrito para $\mathrm{S}_{t} \mathrm{O}_{2}$ ), os grupos de relações fenomenológicas (caixas em azul, numeradas de 1 a 6), e evidencia os desenvolvimentos efetuados pelo nosso grupo: incorporação de um retro-controlo positivo no cálculo do rendimento energético e o cálculo do Q $\mathrm{m}$ com base no V`O _2 (caixas a verde). 
$\mathrm{V} \cdot \mathrm{O} 2 \mathrm{a}$

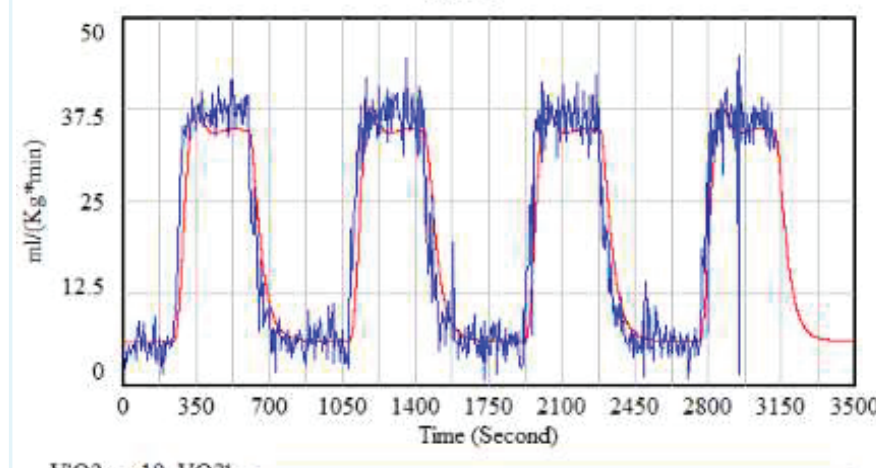

VO2a: 19 VO2k

VO2a:s19_VO2k_sim

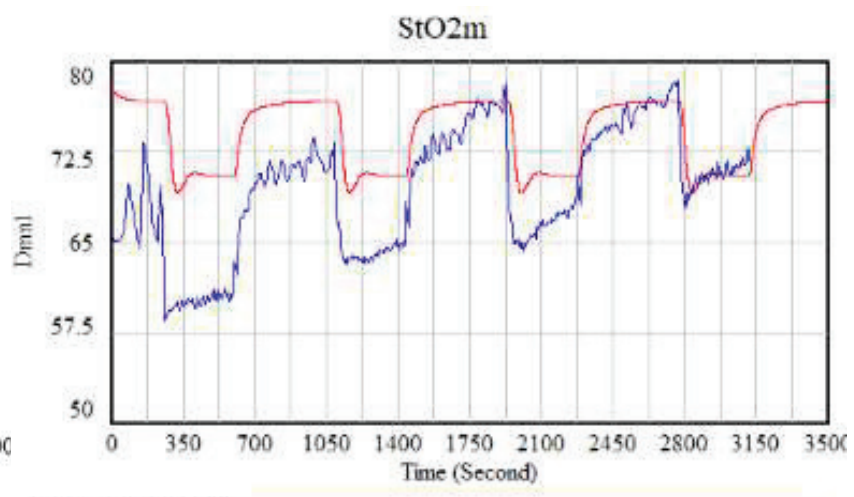

$\mathrm{StO} 2 \mathrm{~m}$ : 519 VO2k

StO2m : s19_VO2k_sim

FIGURA 2. Comportamento experimental (azul) versus simulado (vermelho) durante quatro repetições de exercício de intensidade constante. a) $\mathrm{V} \mathrm{O}_{-} 2$; b) $\mathrm{S}_{t} \mathrm{O}_{2}$.

O diagrama na Fig. 1 apresenta uma versão simplificada do modelo.

Os valores iniciais dos reservatórios são propostos na literatura, nomeadamente:

- Concentração dos fosfatos de alta energia ([ATP PCr]), Mader ${ }^{24}$;

- Conteúdo de oxigénio no leito vascular venoso do músculo e no capilar (O_2 cap) ${ }^{21}$ e conteúdo de oxigénio no sangue venoso em repouso de 15,3 $\mathrm{mL} \cdot \mathrm{dL}-1$;

- Conteúdo de oxigénio no músculo (O_2 tis);

- Concentração de lactato no sangue (C_a Lact);

- Concentração de lactato no músculo (C_m Lact).

As constantes do modelo do peso corporal, percentagem de massa muscular, hematócrito, concentração de

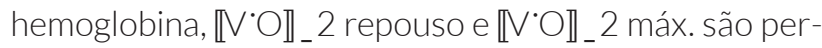
sonalizadas e obtêm-se por medição em laboratório. As outras constantes estão propostas na literatura e são usadas em todas as simulações.

É necessário introduzir no modelo as condições que descrevem o regime de exercício e a altitude:

- Pressão parcial de oxigénio no sangue arterial (P_a O_2), estimada indiretamente pela medição por oximetria de onda de pulso (S_p O_2);

- Potência, W (Watt), obtida por medição com o ciclo ergómetro e que corresponde à intensidade do exercício.

Na Fig. 2 é apresentado um exemplo da confrontação dos resultados medidos com os resultados simulados num exemplo de exercício constituído por quatro repetições de corrida a uma potência determinada.

\section{PERSPETIVAS DE APLICAÇÃO E DESENVOLVIMENTO}

Os modelos são úteis na previsão de cenários. No caso do nosso modelo torna-se possível prever o efeito da alteração de um ou mais parâmetros na resposta fisiológica ao exercício e à altitude e, por isso, é uma ferramenta para a prescrição do exercício mesmo em indivíduos sedentários ou portadores de doenças crónicas.

É desejável alargar o horizonte temporal e incorporar mecanismos que expliquem as alterações na adaptação fisiológica ao esforço e à altitude.

O desenvolvimento de intervenções como novas provas de esforço ou sessões de treino terapêutico podem ser testadas previamente neste modelo. ${ }^{27}$ Segundo Parker e Clermont, ${ }^{28}$ a razão principal do desenvolvimento dos modelos matemáticos é a sua utilização no suporte de decisões em tempo real.

Estes modelos podem ser integrados com outros mode$\operatorname{los}^{29}$ e servir de base para o trabalho de diferentes equipas de investigação, ${ }^{30}$ porque são desenvolvidos com base na conservação de massa, carga, energia e quantidade de movimento, de acordo com as leis da mecânica e da termodinâmica e porque revelam de forma explícita os pressupostos e as aproximações efetuadas.

\section{CONCLUSÃO}

Face ao exposto neste artigo estão criadas as condições para lançamento de um "Núcleo de Investigação Médica" na área da medicina desportiva e da fisiologia que inclua a modelação matemática como ferramenta de investigação. 
CONFLITOS DE INTERESSE: Os autores declaram não ter qualquer conflito de interesse na realização do presente trabalho.

FONTES DE FINANCIAMENTO: Não houve qualquer fonte de financiamento na realização do presente trabalho.

CONFLICTS OF INTEREST: The authors declare that they have no conflicts of interest.

FINANCIAL SUPPORT: This work has not received any contribution, grant or scholarship.

\section{REFERÊNCIAS}

1. Noble D. Claude Bernard, the first Systems Biologist, and the future of Physiology. Exp Physiol. 2008;93:16-26.

2. Bertalanffy LV. General theory of systems - applications to psychology. Soc Sci Inf. 1967;6:125-36.

3. Sterman JD. Learning from evidence in a complex world. Am J Public Health. 2006;96:505-14.

4. Gallaher EJ. Biological system dynamics: from personal discovery to universal application. Simulation. 1996;66:243-57.

5. An G, Faeder J, Vodovotz Y. Translational systems biology: introduction of an engineering approach to the pathophysiology of the burn patient. J Burn Care Res. 2008;29:277-85.

6. Wellstead P, Sreenath S, Cho K. Systems and Control Theory for Medical Systems Biology. In: Daskalaki A, editor. Handbook of Research on Systems Biology Applications in Medicine. Berlin: IGI Global; 2009.

7. Millhorn HT, Benton R, Ross R, Guyton AC. A mathematical model of the human respiratory constrol system. Biophys $J$. 1965;5:27-46.

8. Keener J, Sneyd J. Mathematical Physiology II: Systems Physiology. In: Marsden JE, Sorovich L, editors. Interdisciplinary Applied Mathematics. Berlin: Springer; 2009. p.605.

9. Grodins FS, Buell B. Mathematical analysis and digital simulation of the respiratory control system. J Appl Physiol. 1967;22:260-76.

10. Margaria R. Biomechanics and energetics of muscular exercise. Oxford: Oxford University Press; 1976.

11. Morton RH. On a model of human bioenergetics. Eur J Appl Physiol Occup Physiol. 1985;54:285-90.

12. Wilson DF, Owen CS, Holian A. Control of mitochondrial respiration: A quantitative evaluation of the roles of cytochrome c and oxygen. Arch Biochem Biophys. 1977;182:749-62.

13. Erecińska M, Wilson DF. Homeostatic regulation of cellular energy metabolism. Trends Biochem Sci. 1978;3:219-23.
14. Linnarsson D. The body as a bioenergetic system- lessons from systems engineering and comparative physiology. Med Sci Sports Exerc. 1990;22:59-61.

15. Hughson RL. Exploring cardiorespiratory control mechanisms through gas exchange dynamics. Med Sci Sports Exerc. 1990;22:72-9.

16. Swanson GD. Assembling control models from pulmonary gas exchange dynamics. Med Sci Sports Exerc. 1990;22:80-7.

17. Whipp BJ, Ward SA. Physiological determinants of pulmonary gas exchange kinetics during exercise. Med Sci Sports Exerc. 1990;22:62-71.

18. Barstow TJ, Lamarra N, Whipp BJ. Modulation of muscle and pulmonary $\mathrm{O}_{2}$ uptakes by circulatory dynamics during exercise. J Appl Physiol. 1990;68:979-89.

19. Cabrera ME, Saidel GM, Kalhan SC. Role of $\mathrm{O}_{2}$ in regulation of lactate dynamics during hypoxia: Mathematical model and analysis. Ann Biomed Eng. 1998;26:1-27.

20. Cabrera ME, Saidel GM, Kalhan SC. Lactate metabolism during exercise: analysis by an integrative systems model. Am J Physiol. 1999;277:R1522-36.

21. Lai N, Dash RK, Nasca MM, Saidel GM, Cabrera ME. Relating pulmonary oxygen uptake to muscle oxygen consumption at exercise onset: in vivo and in silico studies. Eur J Appl Physiol. 2006;97:380-94.

22. Lai N, Zhou H, Saidel GM, Wolf M, McCully K, Gladden LB, et al. Modeling oxygenation in venous blood and skeletal muscle in response to exercise using near-infrared spectroscopy. J Appl Physiol. 2009;106:1858-74.

23. Lai N, Camesasca M, Saidel GM, Dash RK, Cabrera ME. Linking pulmonary oxygen uptake, muscle oxygen utilization and cellular metabolism during exercise. Ann Biomed Eng. 2007;35:956-69.

24. Mader A. Glycolysis and oxidative phosphorylation as a function of cytosolic phosphorylation state and power output of the muscle cell. Eur J Appl Physiol. 2003;88:317-38.

25. Moxnes JF, Hausken K, Sandbakk O. On the kinetics of anaerobic power. Theor Biol Med Model. 2012;9:29.

26. Moxnes JF, Sandbakk O. The kinetics of lactate production and removal during whole-body exercise. Theor Biol Med Model. 2012;9:7.

27. Heck H, Schulz H, Bartmus U. Diagnostics of anaerobic power and capacity. Eur J Sport Sci 2003;3:1-23.

28. Parker RS, Clermont G. System engineering medicine: engineering the inflammation response to infectious and traumatic challenges. J R Soc Interface. 2010;7:989-1013.

29. Beard DA, Bassingthwaighte JB, Greene AS. Computational modeling of physiological systems. Physiol Genomics. 2005;23:1-3; discussion 4.

30. Coskun S, Cicek E, Lai N, Dash RK, Ozsoyoglu Z, Ozsoyoglu G. An online model composition tool for system biology models. BMC Syst Biol. 2013;7:88. 